\title{
Stress e qualidade de vida: influência de algumas variáveis pessoais ${ }^{1}$
}

\author{
Maria Angélica Sadir ${ }^{2}$ \\ Márcia Maria Bignotto \\ Marilda Emmanuel Novaes Lipp \\ Pontifícia Universidade Católica de Campinas, Campinas-SP, Brasil
}

\begin{abstract}
Resumo: O stress é um problema de saúde comum tanto no âmbito profissional como no pessoal e possui várias etiologias. $\mathrm{O}$ objetivo deste estudo foi verificar as variáveis pessoais que interferem nos níveis de stress e qualidade de vida de uma amostra de 106 adultos de ambos os sexos que frequentavam uma clínica psicológica. A idade média foi de 38,5 anos. Os participantes foram divididos em categorias profissionais e avaliados com a utilização do Inventário de Qualidade de Vida e o Inventário de Sintomas de Stress de Lipp. Verificou-se que $88 \%$ da amostra apresentam sintomas de stress. A qualidade de vida estava prejudicada, sendo o quadrante de saúde o mais comprometido em todas as pessoas estressadas. Encontrou-se uma associação significativa entre stress e gênero, mas não entre o cargo exercido e o nível de stress aferido. Houve grande compatibilidade entre a autopercepção do stress e seu diagnóstico clínico.
\end{abstract}

Palavras-chave: stress, qualidade de vida, profissões, gênero.

\section{Stress and quality of life: the influence of some personal variables}

\begin{abstract}
Stress is a health problem common both in the professional and personal spheres and has many causes. This study investigated the impact of some personal variables on levels of stress and quality of life in a sample of 106 male and female adults who were attending an outpatient psychology clinic. Mean age average was 38.5 years. Participants were classified according to profession and evaluated through a Quality of Life Inventory and the Lipp Stress Symptom Inventory. Results indicated that $88 \%$ of the participants had high stress level and quality of life was compromised, especially in terms of health. A significant association between stress and gender was found, but not between job position and inferred level of stress. There was compatibility between self-diagnosis of stress and clinical diagnosis.
\end{abstract}

Keywords: stress, quality of life, occupations, gender.

\section{Estrés y calidad de vida: la influencia de algunas variables personales}

Resumen: El estrés es un problema de salud común, tanto en el ámbito profesional como personal e posee varias etiologías. El objetivo de este estudio fué verificar las variables personales que interfieren en los niveles de estrés y calidad de vida en una amuestra de 106 adultos de ambos sexos que frecuentaban una clínica psicológica. La edad media fué de 38,5 años. Los participantes fueron divididos en categorías profesionales y evaluados con la utilización del Inventário de Calidad de Vida y el Inventário de Síntomas de Estres de Lipp. Se verificó que $88 \%$ de la amuestra posee síntomas de estrés. La calidad de vida estaba perjudicada, siendo el cuadrante de salud el más perjudicado en todas las personas estresadas. Se encontró una relación significativa entre estrés y genero pero no entre La profesión ejercida y el nível de estrés evaluado. Hubo gran compatibilidad entre la auto-percepción del estrés y su diagnóstico.

Palabras claves: estrés, calidad de vida, profesiones, genero.

$\mathrm{Na}$ sociedade pós-moderna, o stress tem se tornado um problema de saúde muito comum, atingindo o marco de 40\% na população de São Paulo (Lipp, 2004). Autores como Moraes, Pereira, Lopes, Rocha e Ferreira (2001) acreditam que as mudanças no estilo de vida das pessoas estão deixando-as debilitadas e, com isso, vulneráveis ao stress, que tem assumido o status de doença. No Brasil as pessoas estão cada vez mais estressadas, pois a grande maioria não possui conhecimento de como lidar com suas fontes de tensão (Lipp, 2007).

Segundo definição de Selye (1936), o stress é uma reação do organismo que ocorre frente a situações que exijam

1 Esse texto foi revisado seguindo o Acordo Ortográfico da Língua Portuguesa (1990), em vigor a partir de $1^{\circ}$ de janeiro de 2009.

2 Endereço para correspondência:

Maria Angélica Sadir. Rua Tiradentes, 289, conj. 91. CEP: 13.023-190.

Campinas-SP, Brasil. E-mail: angelicasadir@uol.com.br dele adaptações além do seu limite. Atualmente, os estudos sobre stress abrangem não apenas as consequências no corpo e na mente humana, mas também suas implicações para a qualidade de vida da sociedade. O stress pode afetar a saúde, a qualidade de vida e a sensação de bem-estar como um todo (Lipp, 2001).

As consequências de altos níveis de stress crônico são percebidas pelas licenças médicas e absenteísmo, queda de produtividade, desmotivação, irritação, impaciência, dificuldades interpessoais, relações afetivas conturbadas, divórcios, doenças físicas variadas, depressão, ansiedade e infelicidade na esfera pessoal (Lipp, 2005b). No âmbito do trabalho, as consequências do stress podem incluir depressão, falta de ânimo, falta de envolvimento com o trabalho e a organização, faltas e atrasos frequentes, excesso de visitas ao ambulatório médico e farmacodependência.

O stress emocional tem várias etiologias, podendo se desenvolver devido à necessidade da pessoa de lidar com 
fatores externos ao organismo capazes de criar tensões patológicas. Pode também se desenvolver frente a condições internas capazes de atuar como geradoras de estados tensionais significativos. As fontes internas incluem a ansiedade, o pessimismo, os pensamentos disfuncionais, o padrão de comportamentos de pressa, a competição, a falta de assertividade, entre outros, capazes de gerar um estado de tensão com consequências físicas e psicológicas (Lazarus, 1995).

Os fatores externos muitas vezes estão ligados à ocupação profissional exercida (Oliveira, 2004). Segundo Chiavenato (1999) existem alguns fatores que seriam provocadores do stress no trabalho, como o autoritarismo do chefe, a desconfiança, as pressões e cobranças, o cumprimento do horário de trabalho, a monotonia e a rotina de certas tarefas, o ambiente barulhento, a falta de segurança, perspectiva e progresso profissional e a insatisfação pessoal. Stoner e Freeman (1999) afirmam que um outro estressor pode ser a sobrecarga de papéis, seja quando uma pessoa tem mais trabalho do que pode realizar ou quando não tem habilidades para realizar suas funções. Segundo Lipp (2005b), alguns estressores típicos dos trabalhadores brasileiros são: sobrecarga de trabalho e na família, lidar com a chefia, autocobrança, falta de união e cooperação na equipe, salário insuficiente, falta de expectativa de melhoria profissional e o próprio cargo exercido pela pessoa.

\section{Stress ocupacional}

Um dos meios de sobrevivência do indivíduo é o trabalho. É nele que as pessoas passam a maior parte do seu dia. Por isso a importância de ter suas funções e objetivos pessoais e profissionais bem definidos e bem traçados (Carvalho \& Serafim, 1995). De acordo com Kanaane (1995), o trabalho sempre ocupou lugar central nas comunidades, onde foi sendo limitado pelas condições socialmente estabelecidas. Por meio do trabalho o indivíduo pode se autorrealizar, exercer sua capacidade criativa e estabelecer relacionamentos interpessoais gratificantes. Por outro lado, o trabalho pode também ser fonte de adoecimento quando, por exemplo, contém fatores de risco para a saúde e o trabalhador não dispõe de estratégias para se proteger destes riscos (Murta \& Tróccoli, 2004). Tal situação é capaz de levar ao stress ocupacional, definido como o estado emocional causado por uma discrepância entre o grau de exigência do trabalho e recursos disponíveis para gerenciá-lo (Grandjean, 1998). É produto da relação entre o indivíduo e seu ambiente, sendo que quando as exigências deste ultrapassam a habilidade do trabalhador para enfrentá-las podem acarretar um desgaste excessivo para o organismo, interferindo também na sua produtividade (Perkins, 1995).

Lazarus (1995) propõe que o stress ocupacional ocorre quando o indivíduo avalia as demandas do trabalho como excessivas para os recursos de enfrentamento de que dispõe. Nem todas as pessoas se desgastam com os mesmos estressores; o que determina se o stress irá ou não se instalar é a conjunção do ambiente com as características do indivíduo. De acordo com este autor, as pessoas que têm tendência à depressão, por exemplo, reagiriam mais intensamente aos estressores organizacionais, o que resultaria em problemas como absenteísmo, baixo desempenho e disfunções emocionais.

As várias teorias que conceituam o stress ocupacional como um processo e enfocam seu caráter relacional concordam em considerar as percepções individuais como mediadoras de suma importância do impacto do ambiente de trabalho sobre as respostas do indivíduo (Cooper, Dewe, \& O'Driscoll, 2001; Edwards, 1992; Edwards \& Cooper, 1990; Jex, 1998; Kahn \& Byosiere, 1992; Lazarus, 1995). Paschoal e Tamayo (2004) conceituam o stress ocupacional como um processo em que o indivíduo percebe demandas do trabalho como estressores, os quais, ao exceder sua habilidade de enfrentamento, provocam reações negativas.

Algumas pesquisas enfatizam que existem variáveis que interferem na forma como o indivíduo responde ao stress ocupacional. Além das diferentes ocupações exercidas, a reação ao stress é influenciada por determinadas características pessoais. A vulnerabilidade individual parece ser fator mediador do impacto do estressor na estrutura psicofísica de cada um (Lazarus, 1995).

O stress se tornou mais prevalente nos últimos nove anos, passando de 32\% em 1996 a 35\% em 2005 em pesquisas realizadas com funcionários que não exercem cargos executivos/diretivos, mas que trabalham em escritórios (Lipp, 2005a). O índice de stress entre trabalhadores de fábrica é significativamente menor, com somente $24 \%$ de uma amostra de 350 trabalhadores de fábrica mostrando sinais significativos de tensão excessiva.

$\mathrm{Na}$ literatura científica nacional existem estudos que examinam as relações entre stress e doenças no trabalho em diversos grupos ocupacionais, como burnout em policiais civis (Tamayo \& Tróccoli, 2002) e professores (Codo, 2000), stress e doenças osteomusculares em bancários (Ferreira, 2001; Pinheiro, Tróccoli, \& Paz, 2002), stress e doenças osteomusculares em atletas (Maciel, 1996), stress em enfermeiros (Stacciarini \& Tróccoli, 2002) e em professores (Reinhold, 1996). Estes estudos mostram que o nível de stress difere de acordo com o tipo de ocupação: policiais, controladores de voo e juízes teriam maior índice, jornalistas da mídia diária e bancários viriam em segundo lugar e executivos e atletas em terceiro (Lipp, 2005a).

Segundo Edward e Cooper (1990) existem seis grupos de agentes estressores no trabalho: fatores intrínsecos ao trabalho, papel do indivíduo na organização, relacionamento interpessoal, carreira e realização, estrutura e clima da organização e interface casa/família.

O stress ocupacional tem recebido muita atenção de pesquisadores e também de instituições governamentais. As empresas podem tentar combater o stress por meio de metas realistas, melhoria do ambiente de trabalho e da comunicação, aumento do envolvimento dos funcionários nas decisões, implantação de programas de bem-estar, diminuição de ruídos no local de trabalho, reconhecimento e recompensa aos colaboradores e redução de conflitos pessoais (Chiavenato, 1999). 
Algumas empresas, segundo Wagner III e Hollenbeck (2000), têm adotado o rodízio de cargos (transferência temporária a outra função), em uma tentativa de conceder ao funcionário uma pausa e treiná-lo em várias funções diferentes.

\section{Stress e gênero}

O stress ocupacional atinge tanto homens quanto mulheres, mas alguns estudos têm tentado averiguar se os fatores estressantes os afetam de forma diferente. Um dos primeiros estudos especificamente dedicados à influência do gênero em todas as etapas da reação ao stress foi o Gender and Stress de Barnett, Biener e Baruch (1987).

Miyata, Tanaka e Isuji (1997) realizaram uma pesquisa em um ambulatório com 796 pacientes, na qual estudaram o gênero, o tipo de emprego/ocupação, tipo doença e fatores que poderiam ser causadores de stress. Nos resultados foram encontradas diferenças envolvendo a categoria gênero.

Ainda em Areias e Guimarães (2004), há referência de outro autor, Levi (1999), que constatou em um estudo realizado por ele que alguns grupos apresentavam maior risco para o stress ocupacional; dentre os fatores determinantes para esta ocorrência cita a combinação entre ser mulher e ter excesso de trabalho, uma situação econômica menos favorecida e um inadequado repertório de respostas para enfrentamento de determinadas situações.

Rinaldi (2007) menciona um estudo da Organização Mundial da Saúde (OMS) que revela que tanto em países emergentes como em países desenvolvidos as mulheres no ambiente de trabalho geralmente apresentam maior stress se comparadas aos homens.

Em um estudo que comparava os gêneros na profissão de analistas de sistemas, os autores encontraram que os homens parecem ter mais controle do processo de trabalho, pois referiram maior flexibilidade de horário, bem como o conhecimento do que o resultado do trabalho representa para a população. Percebeu-se que para homens e mulheres este trabalho apresenta a possibilidade de criar, uma vez que não se faz as mesmas tarefas todos os dias. O relacionamento com os colegas apareceu como fator protetor para homens e mulheres. Em relação aos distúrbios psicológicos verificados nesta população, a prevalência de fadiga e dos sintomas de depressão, irritabilidade e ansiedade foi maior entre as mulheres, o que pode estar relacionado a diferenças nas respostas biológicas, nas situações de trabalho, na percepção subjetiva do trabalho em termos de gênero e também na intersecção entre os papéis exercidos pelas mulheres no trabalho e no lar (Rocha \& Debert-Ribeiro, 2001).

No que diz respeito à inter-relação entre os papéis exercidos pelas mulheres no trabalho e em casa, o primeiro aspecto a ser destacado é a superposição de responsabilidades a que a mulher se submete, permanecendo sob sua responsabilidade os cuidados com a casa e a família, paralelamente à participação no mercado de trabalho. No caso das trabalhadoras, tem que se levar em consideração o trabalho doméstico. As exigências deste tipo de trabalho causam desgaste e, somando-se às do trabalho remunerado, convertemse em uma jornada dupla e estressante.

Em seu estudo, Jonathan (2005) objetivou examinar a questão do bem-estar subjetivo e da qualidade de gênero no contexto do empreendedorismo brasileiro. Nesse sentido buscou analisar ganhos e custos psicológicos vivenciados pelas empreendedoras à luz da multiplicidade de papéis que desempenham. No que diz respeito ao espaço pessoal, os resultados indicaram que houve relativamente pouca satisfação em relação à recreação; por outro lado, a saúde suscitou também muita insatisfação em uma parcela das empreendedoras. Os dados demonstraram que estas áreas não estão sendo atendidas adequadamente; parecem estar sendo sacrificadas, necessitando de um resgate que possibilite garantir uma melhor qualidade de vida das mulheres empreendedoras. A autora ainda relatou que duas questões adicionais merecem atenção por envolverem aspectos do universo feminino. A primeira remete à questão da discriminação, e a segunda vem ao encontro da multiplicidade de papéis desempenhados pelas empreendedoras. Algumas empreendedoras experimentaram barreiras devido a seu gênero.

Os papéis relacionados ao trabalho e à família têm diferentes significados para homens e mulheres, dados os múltiplos papéis desempenhados por estas últimas. Essas diferenças podem ser parcialmente responsáveis pelo fato de seus efeitos serem menos benéficos à saúde mental das mulheres quando comparadas aos homens. Acrescenta-se o fato de haver diferenças entre homens e mulheres quanto à natureza e extensão dos conflitos entre trabalho e cuidado dos filhos, das atribuições de responsabilidade aos problemas conjugais, dos sentimentos de culpa e das autoavaliações como pais e cônjuges (Simon, 1995).

O stress pessoal e o stress social podem refletir nas condições de vida fora do âmbito de trabalho e incluem condições de vida caóticas, conflitos na dinâmica familiar e interpessoal e responsabilidades aflitivas. As mulheres que concentram suas energias na carreira sentem-se frequentemente culpadas ou preocupadas com o fato de terem deixado a família de lado. Além disso, o gasto de energia e dedicação para com o marido e filhos pode desviar parte da energia que seria dirigida para a obtenção do sucesso profissional.

\section{Stress e qualidade de vida}

Percebe-se que os efeitos do stress excessivo e contínuo não se limitam ao comprometimento da saúde. O stress pode, além de ter um efeito desencadeador do desenvolvimento de inúmeras doenças, propiciar um prejuízo para a qualidade de vida e a produtividade do ser humano, o que gera um grande interesse pelas causas e pelos métodos de redução do stress.

Segundo Couto (1987) o stress ocupacional interfere na qualidade de vida modificando a maneira como o indivíduo interage nas diversas áreas da sua vida. A relação entre stress e qualidade de vida tem recebido atenção de pesquisadores 
brasileiros, como Lipp e Tanganelli (2002), Guimarães, Landim e Silva (2003), Reinhold (2004) e Pinheiro (2008).

$\mathrm{O}$ conceito de qualidade de vida vem sendo utilizado nos campos da saúde e do trabalho a fim de verificar variáveis presentes nos diversos contextos sociais que possam sofrer intervenção através das políticas de saúde ou de estratégias de gestão empresarial (Souza \& Figueiredo, 2004). Segundo esses mesmos autores, os estudos que se referem aos impactos da organização do trabalho sobre a saúde e qualidade de vida do trabalhador apresentam múltiplas dimensões de análise. Identificar e compreender a determinação do trabalho nas condições de vida constitui um eixo importante para verificar como o trabalhador percebe e organiza suas relações com o mundo "fora do trabalho", contemplando o acesso aos recursos sociais, de educação, saúde, lazer, entre outros.

Qualidade de vida é um estado de bem-estar físico, mental e social e não somente a ausência de doenças. As pessoas que se consideram felizes atribuem sua felicidade ao sucesso em quatro áreas (social, afetiva, saúde e profissional). O pouco tempo dedicado à família em função do alto investimento no trabalho pode acarretar a falta de suporte e apoio quando necessários. Na área social, pode ocorrer o isolamento e a consequente falta de amigos, além do desencadeamento de sérios prejuízos à saúde da pessoa (Lipp, Malagris, \& Novaes, 2007).

Dentro da classe dos problemas de saúde ocupacional, o stress tem sido visto como uma preocupação pelas sérias consequências que pode acarretar para a qualidade de vida do ser humano. Com isso, percebe-se que estudos que identifiquem variáveis pessoais capazes de interferir no nível de stress e na qualidade de vida dos indivíduos são de grande interesse para a literatura, a fim de se enfocar a prevenção secundária da saúde mental e favorecer a produtividade e o bem-estar físico e mental da população.

Com base nessa percepção, o objetivo deste estudo foi verificar, em uma amostra clínica de pessoas que estavam iniciando um tratamento para o stress excessivo, a qualidade de vida, a ocorrência do stress em diferentes cargos ocupacionais, gêneros e estados civis, averiguando se havia associação entre variáveis.

\section{Método}

\section{Participantes}

A amostra do estudo foi escolhida e definida por conveniência, a partir da ordem de procura de atendimento durante o período selecionado. Os participantes foram 106 adultos que procuraram atendimento em uma clínica especializada em tratamento do stress emocional situada no interior do estado de São Paulo, durante um período de seis meses em 2007. Sessenta e um $(57,5 \%)$ eram do sexo masculino e 45 $(42,5 \%)$ do sexo feminino; $60 \%$ eram casados e $40 \%$ solteiros. A idade média apresentada foi de 38,5 anos, variando de 20 a 62 anos de idade. A maior parte dos participantes se dividia em duas faixas etárias: $36 \%$ entre 30 e 39 anos e $33 \%$ entre 40 e 49 anos.
A fim de se verificar uma possível influência do cargo exercido no nível de stress dos participantes, os mesmos foram classificados de acordo com os seguintes cargos: não chefia - escritório (34 pessoas), profissional liberal (33 pessoas), gerente ou supervisor ( 26 pessoas), estudante (7 pessoas) e "do lar" (6 pessoas).

Entre os participantes do cargo de não chefia - escritório, $53 \%$ eram do sexo masculino e $47 \%$ do feminino, sendo que, no geral, $47 \%$ estavam na faixa etária de $30 \mathrm{a}$ 39 anos, $29 \%$ entre 40 e 49 anos e os outros tinham mais de 49 anos, $62 \%$ deles eram casados e $38 \%$ solteiros. Os profissionais liberais dividiram-se entre as faixas etárias de 18 a 29 anos e 40 a 49 anos ( $30 \%$ cada) e 30 a 39 anos (27\%); quatro deles (12\%) tinham entre 50 e 60 anos. Do total de profissionais liberais (18 mulheres e 15 homens), 19 eram casados (58\%) e 14 solteiros (42\%). Dentro da categoria de gerente ou supervisor, $77 \%$ eram homens e $23 \%$ mulheres. Verificou-se que a maioria estava na faixa entre 40 e 49 anos (42\%), e $73 \%$ eram casados. Os estudantes tinham entre 18 e 29 anos de idade, exceto um, que estava na faixa entre 40 e 49 anos; cinco eram do sexo feminino e dois do masculino. Todos eles eram solteiros. Entre as pessoas "do lar", todas eram do sexo feminino, sendo que $50 \%$ tinham entre 30 e 39 anos e $50 \%$ entre 40 e 49 anos. Apenas uma dona de casa não era casada.

\section{Instrumentos}

Utilizaram-se três instrumentos: um questionário para levantamento de dados sociodemográficos, o Inventário de Sintomas de Stress para Adultos (ISSL) (Lipp, 2000) e o Inventário de Qualidade de Vida (Lipp \& Rocha, 1996).

O ISSL permite verificar se a pessoa tem stress, em que fase está (alerta, resistência, quase-exaustão ou exaustão) e qual a prevalência de sintomas, sejam físicos ou psicológicos. Foi validado e padronizado com uma amostra de 1843 adultos. Os respondentes que voluntariamente participaram da avaliação foram recrutados em quiosques montados em shoppings, colégios e universidades; portanto, eram pessoas que estavam atuando regularmente em sua comunidade. A amostra incluiu 1299 respondentes do estado de São Paulo, 352 da Paraíba e 198 do Rio de Janeiro. Sessenta e quatro por cento dos participantes eram do sexo feminino e $35,7 \%$ do masculino. Aplicando a análise de confiabilidade dos itens, obteve-se o coeficiente alfa de 0,9121 , o que significa uma alta confiabilidade do instrumento.

No total, O ISSL inclui 37 itens de natureza somática e 19 de natureza psicológica, totalizando 53 sintomas divididos em três partes, que se referem às fases do stress anteriormente designadas. Em cada uma das partes a pessoa deve assinalar os sintomas físicos ou psicológicos das últimas 24 horas, da última semana ou do último mês, respectivamente. A sequência dos itens que compõem o inventário segue uma ordem hierárquica de intensidade de sintomas, pois é comum a sintomatologia aparecer na fase de alerta, com pequena intensidade ou frequência, desaparecer na fase de resistência 
(houve o enfrentamento ou adaptação ao agente estressor) e reaparecer na fase de quase-exaustão ou exaustão com maior intensidade (Lipp \& Malagris, 1995).

A fase de alerta é considerada a fase positiva do stress. $\mathrm{O}$ organismo produz adrenalina, que gera energia e vigor na pessoa. Se o stress continuar presente pode-se chegar à fase de resistência, em que a pessoa tenta lidar com os seus estressores de modo a manter sua homeostase interna. A terceira fase (quase-exaustão) é caracterizada pelo enfraquecimento do organismo, que não está mais conseguindo se adaptar ou resistir ao fator estressor. Nesta fase, o processo de adoecimento se inicia e os órgãos que possuírem maior vulnerabilidade genética ou adquirida passam a mostrar sinais de deterioração. Na fase de exaustão, a fase final do stress, podem ocorrer doenças graves (Lipp \& Malagris, 1995).

O Inventário de Qualidade de Vida avalia quatro áreas: social, afetiva, profissional e saúde. Este instrumento ainda se encontra em processo de validação. Contém 45 itens divididos em dez de cada área, sendo que na área de saúde são 15 itens. A pessoa deve assinalar se o que está descrito corresponde à sua realidade. Somam-se os pontos obtidos e, através de um escore, determina-se se tem sucesso ou fracasso em cada área. Só é considerado que a pessoa tem uma boa qualidade de vida quando alcança sucesso nos quatro quadrantes.

\section{Procedimento}

Os instrumentos foram aplicados por uma psicóloga da clínica durante as duas primeiras sessões terapêuticas, como parte da avaliação de stress usualmente conduzida no tratamento do stress oferecido. Os participantes responderam verbalmente aos instrumentos e assinaram um Termo de Consentimento Livre e Esclarecido permitindo que os dados fossem divulgados para fins científicos, anonimamente.

\section{Análise dos dados}

A análise de dados utilizada primeiramente incluiu os percentuais para a caracterização quanto aos dados biográficos dos participantes. Para comparação das variáveis categóricas foi utilizado teste exato de Fisher, devido à presença de valores esperados menores que 5 . O nível de significância adotado para os testes estatísticos foi de $5 \%$, ou seja, $p<0,05$.

\section{Resultados}

A avaliação dos resultados obtidos no ISSL revelou que a grande maioria dos participantes da pesquisa (88\%) recebeu o diagnóstico de stress. Todas as mulheres do estudo e $79 \%$ dos homens tiveram esse diagnóstico. Das 13 pessoas sem stress, que representaram somente $12 \%$ da amostra, todos eram homens. Encontrou-se o predomínio de sintomas psicológicos em todos os cargos pesquisados.
A Tabela 1 apresenta os resultados quanto ao diagnóstico de stress, faixa etária e estado civil, por cargo ocupacional, sendo que os participantes foram classificados nas categorias de não chefia - escritório (32\%), profissional liberal $(31 \%)$, Gerente ou supervisor $(25 \%)$, estudante $(6 \%)$ e "do lar" $(6 \%)$.

Tabela 1

Categorização da amostra por cargo ocupacional.

\begin{tabular}{lcccc}
\hline \multicolumn{1}{c}{ Cargo } & $\begin{array}{c}\text { Stress } \\
\text { \% }\end{array}$ & $\begin{array}{c}\text { Faixa etária } \\
\text { predominante } \\
\text { (anos) }\end{array}$ & \multicolumn{2}{c}{ Estado civil (\%) } \\
Solteiros & Casados \\
\hline Estudante & 100 & $18-29$ & 100 & - \\
“Do lar" & 100 & $30-39$ & 17 & 83 \\
& & $40-49$ & & \\
Profissional & 91 & $18-29$ & 42 & 58 \\
liberal & & $40-49$ & & \\
$\begin{array}{l}\text { Não chefia }- \\
\text { escritório }\end{array}$ & 85 & $30-39$ & 38 & 62 \\
$\begin{array}{l}\text { Gerente ou } \\
\text { supervisor }\end{array}$ & 81 & $40-49$ & 27 & 73 \\
\hline
\end{tabular}

Todas as mulheres no grupo "do lar" estavam com stress, assim como todos os estudantes avaliados. Pode-se verificar na Tabela 1 que $91 \%$ dos profissionais liberais apresentavam stress, sendo 18 homens (60\%) e 12 mulheres (40\%). Entre os participantes do cargo de não chefia - escritório, $85 \%$ apresentaram stress, sendo que dos estressados 13 eram homens (45\%) e 16 mulheres (55\%). Dentro da categoria de gerente ou supervisor, $81 \%$ estavam com stress elevado, sendo $71 \%$ do sexo masculino e $29 \%$ do feminino.

Por meio da análise estatística aplicada verificou-se não haver associação significativa entre apresentar ou não stress e o cargo ocupacional ocupado, considerando-se gerentes, não gerentes e profissionais liberais $\left(\chi_{\mathrm{gl}=2}^{2}=1,267, p=0,53\right)$.

Ao se comparar as variáveis stress e idade nas diferentes categorias profissionais, percebe-se que, exceto na categoria estudantes, a maior incidência de stress está na faixa etária entre 40 e 49 anos; na de escritório - não chefia, 52\% estavam entre 40 e 49 anos. A análise estatística efetuada pelo Teste Exato de Fisher mostrou não haver associação significativa entre as faixas etárias, níveis de stress e cargo ocupado ( $p=0,2356)$.

Quanto à associação entre o stress e o gênero, a análise estatística realizada pelo Teste Qui-quadrado demonstrou ser ela muito significativa $\left(\chi_{\mathrm{gl}=1}^{2}=9,040, p=0,0026\right)$. Um número maior de homens apresentou stress nos cargos de gerente ou supervisor $(71 \%)$ e profissional liberal $(60 \%)$, conforme revela a Tabela 2. Já nas mulheres, esta incidência foi maior nos cargos de escritório - não chefia $(55 \%)$ e quando eram estudantes $(71 \%)$. 
Tabela 2

Percentagens de sucesso e fracasso em cada quadrante da qualidade de vida em pessoas com stress por cargo exercido.

\begin{tabular}{|c|c|c|c|c|c|c|c|c|}
\hline \multirow{3}{*}{ Cargo } & \multicolumn{6}{|c|}{ Qualidade de Vida } & & \\
\hline & \multicolumn{2}{|c|}{ Afetivo (\%) } & \multicolumn{2}{|c|}{ Profissional (\%) } & \multicolumn{2}{|c|}{ Social (\%) } & \multicolumn{2}{|c|}{ Saúde (\%) } \\
\hline & Sucesso & Fracasso & Sucesso & Fracasso & Sucesso & Fracasso & Sucesso & Fracasso \\
\hline Gerente ou supervisor & 29 & 71 & 57 & 43 & 38 & 62 & 19 & 81 \\
\hline Não chefia - escritório & 69 & 31 & 31 & 69 & 79 & 21 & 21 & 79 \\
\hline Estudante & 29 & 71 & 29 & 71 & 71 & 29 & - & 100 \\
\hline "Do lar" & 67 & 33 & 17 & 83 & 67 & 33 & - & 100 \\
\hline Profissional liberal & 43 & 57 & 70 & 30 & 50 & 50 & 3 & 97 \\
\hline
\end{tabular}

Os participantes casados contaram com maior representação na maioria dos cargos exercidos: gerente ou supervisor (67\%), escritório - não chefia (59\%), dona de casa (83\%) e profissional liberal (57\%). Como todos os estudantes eram solteiros, não se pode fazer essa comparação para o grupo. A análise realizada pelo Teste Exato de Fisher demonstrou que a diferença entre os participantes casados e solteiros foi significativa $(p=0,04)$.

Em relação à qualidade de vida, nos participantes com stress, verificou-se que em todos os cargos o quadrante saúde foi o mais prejudicado. $\mathrm{O}$ cargo de supervisores e gerentes foi a categoria que significativamente obteve mais prejuízo no quadrante social, enquanto o menor prejuízo foi para profissionais liberais $\left(\chi_{\mathrm{gl}=2}^{2}=9,57, p=0,008\right)$.

Os profissionais liberais tiveram maior sucesso no quadrante afetivo, ao passo que, novamente, supervisores e gerentes apresentaram o maior prejuízo $\left(\chi^{2}{ }_{\mathrm{gl}=2}=8,51, p=0,01\right)$. Nos quadrantes profissional $\left(\chi_{\mathrm{gl}=2}^{2}=2,58, p=0,27\right)$ e de saúde $\left(\chi_{\mathrm{gl}=2}^{2}=4,41, p=0,10\right)$ essa associação não foi significativa.

\section{Discussão}

$\mathrm{Na}$ amostra estudada, houve um alto nível de stress ao se comparar com o índice de stress relatado por Lipp (2005a), o que era esperado uma vez que a amostra estudada se constituiu de pessoas que procuraram tratamento especificamente para o stress. O alto nível de stress encontrado, portanto, demonstra a compatibilidade entre a autopercepção do stress e o seu diagnóstico, visto que $88 \%$ das pessoas que procuraram um serviço especializado para tratamento em uma clínica de stress tiveram uma percepção correta do seu diagnóstico.

A falta de correlação encontrada entre níveis de stress e cargos ocupacionais não corresponde a alguns dados encontrados na literatura. Segundo Oliveira (2004) alguns fatores externos ao stress estão ligados à ocupação profissional exercida. Lipp (2004, 2005b), afirma que várias profissões já foram estudadas e conclui que diferentes ocupações geram diferentes níveis de stress. Nos resultados de suas pesquisas, o índice de stress entre trabalhadores de fábrica é significativamente menor que o de outros níveis de trabalhadores, demonstrando que há diferenças entre as ocupações.
O estudo realizado sobre fatores de stress em diferentes categorias profissionais, realizado por Chan, Lai, Ko e Kam (2000) mostrou que os profissionais com melhor qualificação e principalmente que exercem maior autonomia e controle sobre o processo de trabalho apresentaram melhores condições de enfrentamento de situações causadoras de desgaste mental.

No estudo aqui descrito o fato de esta diferença não ser significativa pode ser explicado com base em alguns estudos que enfatizam existirem algumas variáveis que interferem na forma como o indivíduo responde ao stress ocupacional. As percepções individuais do impacto do ambiente de trabalho sobre as respostas do indivíduo podem ser mediadoras do stress (Cooper, Dewe, \& O’Driscoll, 2001; Edwards, 1992; Edwards \& Cooper, 1990; Jex, 1998; Kahn \& Byosiere, 1992; Lazarus, 1995).

O fato de que não se encontrou diferença significativa na prevalência de stress entre os cargos pode dar origem a duas hipóteses: (a) visto que a maior parte da amostra estava estressada, o fator saturação não permitiu ver as diferenças entre os cargos; e (b) o stress presente nos participantes poderia ser oriundo de outras fontes que não aquelas ligadas ao trabalho.

Outros dados obtidos dão suporte à literatura existente. Os resultados indicaram uma relação muito significativa entre stress e gênero, tendo-se verificado que as mulheres se encontravam com maiores níveis de stress, o que condiz com Rinaldi (2007), que menciona que tanto em países emergentes quanto em países desenvolvidos as mulheres geralmente apresentam maior stress, se comparadas aos homens, no ambiente de trabalho. Barnett, Marshall, Raudenbush e Brennan (1993) relataram que, devido a um número crescente de mulheres incorporado à população ativa e ocupando postos de trabalhos tradicionalmente desempenhados por homens, aumenta a necessidade de analisar a influência do gênero na relação entre doença e stress no trabalho, por estas serem expostas a muitas fontes estressoras.

Autores como Areias e Guimarães (2004) demonstraram que pelo fato de as mulheres ficarem sujeitas a uma dupla sobrecarga de trabalho ocorre um dilema comum entre casamento, filhos e demandas ocupacionais, o que poderia contribuir para um nível elevado de stress. Outros fatores 
citados pelos estudos como fonte de stress ocupacional em mulheres remete à questão da discriminação e à multiplicidade de papéis desempenhados por elas (Jonathan, 2005).

Levi (1999) afirma que, além das variáveis citadas acima, a combinação entre ser mulher e ter excesso de trabalho, uma situação econômica menos favorecida e um inadequado repertório de respostas para enfrentamento de determinadas situações faz com que as mulheres apresentem um maior nível de stress do que os homens, como ocorreu na amostra estudada.

Outra correlação encontrada foi entre stress e estado civil: os profissionais casados apresentaram maiores níveis de stress, o que vem a ser coerente com a literatura, que postula que a quantidade de número de papéis sociais está relacionada a maiores chances de níveis de stress (Rocha \& DebertRibeiro, 2001). Percebe-se que após o casamento, tanto os homens quanto as mulheres assumem mais papéis e, com isso, responsabilidades, tanto de cuidado com a casa e com os filhos, quanto no que diz respeito à questão financeira, o que pode gerar mais conflitos e autoavaliações negativas por parte de ambos (Simon, 1995).

Quanto à qualidade de vida avaliada no estudo os dados observados demonstram que a qualidade de vida, de forma geral, estava prejudicada nos participantes, como sugere Lipp (2005b) quando relata que as consequências de altos níveis de stress crônico são percebidas pelas licenças médicas e absenteísmo, queda de produtividade, desmotivação, irritação, impaciência, dificuldades interpessoais, relações afetivas conturbadas, divórcios, doenças físicas variadas, depressão, ansiedade e infelicidade no ambiente pessoal. Segundo Couto (1987), o stress ocupacional interfere na qualidade de vida modificando a maneira como o indivíduo interage nas diversas áreas da sua vida.

Dentre os quadrantes que constituem a qualidade de vida, o quadrante de saúde foi o mais prejudicado em todos os cargos ocupacionais. Os autores Lipp, Malagris e Novaes (2007) afirmam que o stress pode desencadear sérios prejuízos na saúde da pessoa. Houve correlações entre o cargo ocupado e o sucesso na área específica, sendo que nas áreas social e afetiva os profissionais liberais apresentaram maiores sucessos nessas categorias, enquanto para os supervisores e gerentes os prejuízos foram maiores. No estudo realizado por Souza e Figueiredo (2004) pode-se perceber que o nível de qualificação profissional, considerando o conjunto de seus determinantes, configurou um importante indicador das diferenças encontradas na percepção da qualidade de vida, quanto aos aspectos de saúde e autonomia para o enfrentamento do contexto de vida.

Esses dados tornam-se relevantes por demonstrarem que devido ao fato de o profissional liberal ter a possibilidade de ser mais independente quanto à estrutura de sua carga de trabalho, talvez tenha mais possibilidades de cuidar de sua qualidade de vida, usufruindo de maior tempo para suas atividades sociais e para se dedicar à sua vida familiar e afetiva.

\section{Considerações finais}

O estudo realizado tem implicações para o campo da psicologia organizacional, em especial na área da saúde mental, enfocando a prevenção secundária.

Houve correspondência entre a autopercepção de stress, que deu origem à procura de uma clínica especializada no tratamento do stress, e o diagnóstico clínico realizado por meio de teste padronizado e validado, que revelou altos níveis de stress na amostra. O fato de que não se encontrou diferença significativa na prevalência de stress entre os cargos pode ser atribuído ao nível alto de stress presente em quase todos os participantes, o que tornaria difícil detectar diferenças entre os cargos. Outra possibilidade seria a de o stress presente nos participantes ser oriundo de outras fontes que não aquelas ligadas ao trabalho.

Outro fato observado diz respeito à qualidade de vida de pessoas que possuem cargos ocupacionais diferenciados: profissionais liberais apresentaram bons resultados nas áreas social e afetiva, ao contrário do encontrado entre os supervisores e gerentes que tiveram essas mesmas áreas como as mais prejudicadas. Este dado certamente merece maiores investigações.

A implantação de programas que promovam condições para a promoção da saúde física e emocional das pessoas, como a atenção que deve ser dada ao stress, tendo em vista todas as suas possíveis consequências ao ser humano descritas no decorrer deste estudo, mostra-se benéfica e necessária.

Foram detectados aspectos que devem ser mencionados como relevantes por evidenciarem como o stress organizacional pode interferir na qualidade de vida das pessoas. Esta interferência pode trazer sérios prejuízos para quase todas as áreas, como foi constatado no campo da saúde física, que demonstrou estar prejudicada em todas as pessoas estressadas da amostra. Tal dado é de fundamental importância e merece atenção das organizações, tendo em vista que, para que haja uma boa produtividade, o funcionário deve possuir uma saúde adequada.

Outro dado importante que mostrou interferir na qualidade de vida de pessoas que possuem cargos ocupacionais diferenciados foi que profissionais liberais apresentaram bons resultados nas áreas social e afetiva, podendo-se levantar a hipótese de que um profissional liberal possa ter maior controle quanto à organização de seu horário, o que lhe possibilita um equilíbrio maior entre algumas áreas de sua vida, ao contrário do encontrado entre os supervisores e gerentes que tiveram essas áreas (social e afetiva) como as mais prejudicadas.

Nesta pesquisa percebeu-se a importância de se avaliar tanto o stress e a qualidade de vida quanto as variáveis que interferem nesses aspectos, a fim de se implementar programas que promovam condições para a promoção da saúde física e emocional das pessoas, como a atenção que deve ser dada ao stress, tendo em vista todas as possíveis consequências ao ser humano descritas no decorrer deste estudo. 


\section{Referências}

Areias, M. E. Q., \& Guimarães, A. M. (2004). Gênero e estresse em trabalhadores de uma universidade pública do Estado de São Paulo. Psicologia em estudo, 9, 255-262.

Barnett, R. C., Biener, L., \& Baruch, G. K. (1987). Gender and stress. Nueva York: Free Press.

Barnett, R. C., Marshall, N. L., Raudenbush, S. W., \& Brennan, R. T. (1993). Gender and the relationship between job experiences and psychological distress: A study of dual-earner couples. Journal of Personality and Social Psychology, 64, 794-806.

Carvalho, A. V., \& Serafim, O. C. G. (1995). Administração de recursos humano (Vol. 2). São Paulo: Pioneira.

Chan, B. K., Lai, G., Ko, Y. C., \& Kam, W. B. (2000). Work stress among six professional groups: The Singapore experience. Social Science \& Medicine, 50, 1415-1432.

Chiavenato, I. (1999). Gestão de Pessoas: O novo papel dos recursos humanos nas organizações. Rio de Janeiro: Campus.

Codo, W. (Org.). (2000). Educação: Carinho e trabalho. Petrópolis: Vozes.

Cooper, C. L., Dewe, P. J., \& O’Driscoll, M. P. (2001). Organizational stress: A review and critique of theory, research and applications. London: Sage Publications Edwards.

Couto, H. A. (1987). Stress e qualidade de vida dos executivos. Rio de Janeiro: COP.

Edwards, J. R. (1992). A cybernetic theory of stress, coping, and well-being in organizations. The Academy of Management Review, 17, 238-274.

Edwards, J. R., \& Cooper, C. L. (1990). The personenvironment fit approach to stress: Recurring problems and some suggested solutions. Journal of Organizational Behavior, 11, 293-307.

Ferreira, M. C. P. (2001). Bancários portadores de distúrbios osteomusculares relacionados ao trabalho e identidade profissional. Estudos, 28, 749-781.

Grandjean, E. (1998). Manual de Ergonomia: Adaptando o trabalho ao homem (4a ed.). Porto Alegre: Bookman.

Guimarães, M. C., Landin, L. de S., \& Silva, H. R. (2003). Estresse ocupacional e sofrimento no trabalho: Um estudo com caminhoneiros. Revista de Psicologia (Fortaleza), 21(1/2), 54-63.

Jex, S. M. (1998). Stress and job performance. London: Sage Publications.

Jonathan, E. G. (2005). Mulheres empreendedoras: Medos, preocupações e qualidade de vida. Psicologia em Estudo, 10, 373-382.

Kahn, R., \& Byosiere, P. (1992). Stress in organizations. In M. A. Dunnette \& L. M. Hough, Handbook of industrial \& organizational psychology (Vol. 3, pp. 571-650). Palo Alto: Consulting Psychologists Press.
Kanaane, R. (1995). Comportamento humano nas organizações: O homem rumo ao século XXI. São Paulo: Atlas.

Lazarus, R. S. (1995). Psychological stress in the workplace. In R. Crandall \& P. L. Perrewé (Orgs.), Occupational stress: A handbook (pp. 3-14). Washington, USA: Taylor \& Francis.

Levi, L. (1999). Guía sobre el estrés relacionado con el trabajo: La "sal de la vida" o el "beso de la muerte?". Barcelona: Dirección General de Empleo y Asuntos Sociales de la Comisión Europea de Seguridad y Salud en el Trabajo.

Lipp, M. E. N. (2000). Inventário de Sintomas de Stress para Adultos. São Paulo: Casa do Psicólogo.

Lipp, M. E. N. (Org.). (2001). Pesquisas sobre stress no Brasil: Saúde, ocupações e grupos de risco. Campinas: Papirus.

Lipp, M. E. N. (Org.). (2004). Stress no Brasil: Pesquisas avançadas. Campinas: Ed. Papirus.

Lipp, M. E. N. (2005a). Stress e o turbilhão da raiva. Campinas: Casa do Psicólogo.

Lipp, M. E. N. (2005b). Stress no trabalho: Implicações para a pessoa e para a empresa. In F. P. N. Sobrinho, \& I. Nassaralla, Pedagogia Institucional: Fatores humanos nas organizações. Rio de Janeiro: Zit.

Lipp, M. E. N. (2007). Globalização e mudanças: O stress do novo milênio. Recuperado em 10 janeiro 2007, de http://www.estresse.com.br

Lipp, M. E. N., \& Rocha, J. C. (1996). Stress, qualidade de vida e hipertensão arterial. Campinas: Papirus.

Lipp, M. E. N., \& Tanganelli, M. S. (2002). Stress e qualidade de vida em magistrados da justiça do trabalho: Diferenças entre homens e mulheres. Psicologia: Reflexão e Crítica, $15,537-48$

Lipp, M. E. N., Malagris, L. E. N., \& Novais, L. E. (2007). Stress ao longo da vida. São Paulo: Ícone.

Lipp, M. E. N., \& Malagris, L. E. N. (1995). Manejo do estresse. In B. Rangé (Ed.). Psicoterapia comportamental e cognitiva: pesquisa, prática, aplicação e problemas (pp. 279-92). Campinas: Psy II.

Maciel, S. V. (1996). Atleta juvenil feminina: Correlação entre características psicológicas, stress e lesões osteomusculares. In M. E. N. Lipp (Org.), Pesquisas sobre stress no Brasil: Saúde, ocupações e grupos de risco (pp. 211-224). Campinas: Papirus.

Miyata, M., Tamaka,Y., \& Tsuji, S. (1997). Occupational stress as the cause of psychosomatic and mental disorders. Sangyo Ilka Daugaku Zasshi, 19(4), 297-305.

Moraes, L. F. R., Pereira, L. Z., Lopes, H. E. G., Rocha, D. B., \& Ferreira, S. A. A. (2001). Estresse e qualidade de vida no trabalho na polícia militar do estado de Minas Gerais. Anais do Encontro da Associação Nacional de Pós-graduação e Pesquisa em Administração, 24, 4-28. 
Murta, S. G., \& Tróccoli, B. T. (2004). Avaliação de intervenção em estresse ocupacional. Psicología: Teoria e Pesquisa, 20, 39-47.

Oliveira, J. B. (2004). Fontes e sintomas de stress em juízes e servidores públicos: Diferenças entre homens e mulheres. Dissertação de Mestrado não-publicada, Pontifícia Universidade Católica de Campinas, Campinas, SP.

Paschoal, T., \& Tamayo, A. (2004). Impacto dos valores laborais e da interferência família- trabalho no estresse ocupacional. Psicologia: Teoria e Pesquisa, 21, 173180.

Perkins, V. (1995). Stress: O ponto de ruptura. São Paulo: Jovens Médicos.

Pinheiro, C. R. (2008). Stress e qualidade de vida em clérigos(as). Dissertação de Mestrado não-publicada, Pontifícia Universidade Católica de Campinas, Campinas, SP.

Pinheiro, F. A., Tróccoli, B., \& Paz, M. G. T. (2002). Aspectos psicossociais dos distúrbios osteomusculares (Dort/LER) relacionados ao trabalho. In A. M. Mendes, L. O. Borges, \& M. C. Ferreira (Orgs.), Trabalho em transição, saúde em risco (pp. 65-85). Brasília: Editora Universidade de Brasília.

Reinhold, H. H. (1996). Stress ocupacional do professor. In M. E. N. Lipp (Org.), Pesquisas sobre stress no Brasil: Saúde, ocupações e grupos de risco (pp.169-194). Campinas: Papirus.

Reinhold, H. H. (2004). O sentido da vida: Prevenção de stress e burnout do professor. Tese de Doutorado não-publicada, Pontifícia Universidade Católica de Campinas, SP.

Rinaldi, A. (2007). OMS coloca o estresse ocupacional como um fator social. Recuperado em 11 abril 2007, de http:// www.fundacentro.gov.br/CTN/noticias.asp?Cod $=534$

Rocha, L. E., \& Debert-Ribeiro, M. (2001). Trabalho, saúde e gênero: Estudo comparativo sobre analistas de sistemas. Revista de Saúde Pública, 35, 539-547.

Selye, H. A. (1936). Syndrome produced by diverce nervous agents. Nature, 138, 32.

Simon, R. W. (1995). Gender, multiple roles, role meaning, and mental health. Journal of Health Social Behavior, 36, 182-194.

Souza, L. B., \& Figueiredo, M. A. C. (2004). Qualificação profissional e representações sobre trabalho e qualidade de vida. Paidéia (Ribeirão Preto), 14, 221-232.

Stacciarini, J. M. R., \& Tróccoli, B. (2002). Stress ocupacional. In A. M. Mendes, L. O. Borges, \& M. C. Ferreira (Orgs.), Trabalho em transição, saúde em risco (pp. 185-205). Brasília: Editora Universidade de Brasília.

Stoner, J. A. F., \& Freeman, R. E. (1999). Administração (5a ed.). Rio de Janeiro: LTC.

Tamayo, M. R., \& Tróccoli, B. (2002). Burnout no trabalho. In A. M. Mendes, L. O. Borges, \& M. C. Ferreira (Orgs.), Trabalho em transição, saúde em risco (pp. 43-58). Brasília: Editora Universidade de Brasília.

Wagner III, J. A., \& Hollenbeck, J. R. (2000). Comportamento organizacional. São Paulo: Saraiva.
Maria Angélica Sadir é doutoranda em Psicologia pelo Programa de Pós-graduação Stricto Sensu do Centro de Ciências da Vida da Pontifícia Universidade Católica de Campinas, bolsista CAPES.

Márcia Maria. Bignotto é doutoranda em Psicologia pelo Programa de Pós-graduação Stricto Sensu do Centro de Ciências da Vida da Pontifícia Universidade Católica de Campinas, bolsista CAPES.

Marilda Emmanuel Novaes Lipp é Professora Doutora da Faculdade de Psicologia da Pontifícia Universidade Católica de Campinas.

Recebido: $18 / 05 / 2008$

$1^{a}$ revisão: 04/03/2009

$2^{a}$ revisão: $18 / 06 / 2009$

Aceite final: 26/06/2009 